\title{
Feature geometry in disordered phonologies
}

\author{
STEVEN B. CHIN and DANIEL A. DINNSEN
}

Department of Linguistics, Indiana University, Bloomington, IN 47405, USA

(Received 29 January 1991; accepted 8 May 1991)

\begin{abstract}
Two types of systems are in general use for the description and classification of consonants in disordered phonological systems: conventional place-voice-manner and standard distinctive features. This paper proposes the use of a third model, feature geometry, which is an analysis framework recently developed in the linguistic study of primary languages. Feature geometry allows for relatively independent behaviour of individual distinctive features, but also organizes them into hierarchies in order to capture the fact that features very often act together in rules. Application of the feature geometry to the study of the phonologies of 40 misarticulating children, specifically to the phenomena of apparent cluster coalescence, fricative/affricate alternations, and alveolar stop/glottal stop alternations, reveals that feature geometry provides better explanations for representations and rules in disordered systems than either of the other two frameworks.
\end{abstract}

Keywords: Feature geometry, distinctive features, functional misarticulation, phonological disorders.

\section{Introduction}

Two types of systems are in general use for the description and classification of consonants in disordered phonological systems. These are first, systems using place of articulation, voicing, and manner of articulation; and second, systems using distinctive features. The choice of using one or the other type of description is often more a matter of training tradition rather than true theoretical inclination. However, the systems are not specifically interchangeable, and their theoretical predictions can evidence differences that extend into the realm of phonological remediation, so that their advantages and disadvantages can have implications for descriptions of phonologies and for any assessment and treatment depending on such descriptions. A recent idea in linguistic theory is feature geometry, an extension of generative phonology that groups distinctive features hierarchically and overcomes some of the difficulties inherent in using either place-voice-manner or standard distinctive features systems exclusively.

Modern systems using place-voice-manner are based on the principles set forth by the International Phonetic Association, and specifically on the summary of principles implicit in its alphabet chart. The most recent version from 1989 (see Ladefoged, 1990 or Duckworth, Allen, Hardcastle, and Ball, 1990) maintains the tradition of arranging alphabetic symbols for consonants in a grid, with each cell defined as the intersection of place of articulation and manner of articulation. Each cell can further contain the symbols for both a voiceless and a voiced sound. Groups 
of consonants can be defined by reference to an entire row or column on the grid, such as "the fricatives" or "the velars". However, there is no explicit mechanism for simultaneous reference to more than one row or column. One must resort to conjunctive expressions like 'the labials and the dentals' or 'the nasals, the trills, and the lateral approximants". Further. the necessary use of these periphrastic devices does not prohibit on principled grounds the grouping of sounds that do not appear to act together in any system yet investigated. for instance. "the plosives and the palatals' or 'the fricatives and the labiodentals".

Distinctive feature systems. on the other hand, allow for the description of both any single manner or place of articulation and also groups of manners or places that do act together in phonological systems. Further, it is a theoretical requisite, if not always a practical success, that feature systems be prohibited from describing a sound or group of sounds that is impossible or at least unattested. Modern feature systems allow each feature to act independently of all others. as evidenced by the practice of simply listing features and their coefficients in random order in a matrix. We know. however. that features often do act in concert. For instance, the feature system suggested by Chomsky and Halle (1968) requires the use of two features, [anterior] and [coronal], to describe any single consonantal place of articulation. But the theory itself has no way to guarantee or require that these features act together. On the other hand, there is also no theoretical way to prohibit merely random groups of features from acting together.

\section{Feature geometry}

Recently, researchers in the phonologies of primary languages have extended the findings of autosegmental phonology (see Goldsmith. 1990) and developed a method called feature geometry, whereby broad categories such as 'place of articulation' and narrower categories such as "coronal" are reconciled in a single descriptive system by organizing features into a hierarchical tree. In this way, feature geometry is able to reconcile the broad categories of place-voice-manner systems and the finergrained analytical units of distinctive feature systems. This reconciliation is achieved by grouping features that behave together, for instance, the place features, under single dominating nodes. For instance. (1) shows that part of the feature geometry that defines place of articulation. (As is the case with many linguistic models, feature geometry is accompanied by graphic representations to clarify and illuminate its concepts. Note, however, that the relative value or strength of a model or theory is to be sought more in the concepts themselves than in their graphic representation.)

(1) Expansion of place

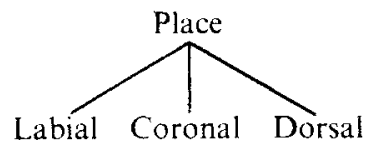

In (2), we see the feature geometry suggested by Sagey (1986), but other configurations have been suggested, for instance by Clements (1985) and McCarthy (1988).

As in autosegmental phonology, temporal sequencing is achieved in feature geometry by arraying like nodes on the same level in the hierarchy on a tier. In 
(2) Feature geometry from Sagey (1986)

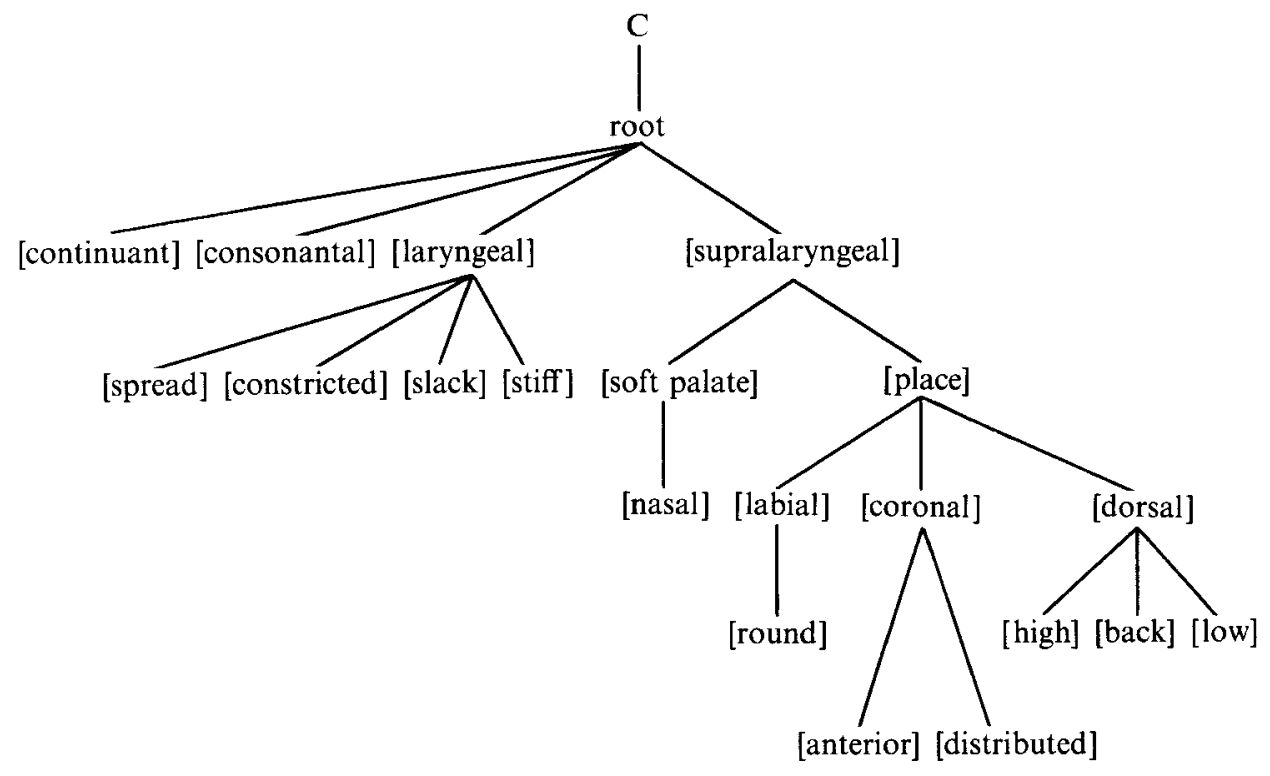

Sagey's (1986) model, the topmost tier in a feature geometry is a 'skeletal tier', which contains two types of nodes: first Cs, which represent non-syllabic segments, and second, Vs, which represent syllabic segments. Each C or V dominates a 'root node', which is the structural representation of a phoneme. Each root node dominates two binary-valued feature nodes, [continuant] and [consonantal], and two class nodes, [laryngeal] and [supralaryngeal]. The laryngeal node dominates features that define, for instance, the difference between 'voiced' sounds and 'voiceless' ones. The supralaryngeal node dominates two further class nodes: first, [soft-palate], whose presence or absence determines the difference between nasal and non-nasal segments, and second, the [place] node, which dominates the articulator nodes [labial], [coronal], and [dorsal]. Finally, the articulator class nodes dominate binary-valued features that specify how each articulator is used.

Immediately adjacent tiers form a plane, across which nodes are connected by 'association lines', which indicate temporal overlap. As illustrated in (3), the total structure of nodes and features joined by association lines and ultimately dominated by the root node define the character of a segment. For instance, the structure in (3) is the representation for the segment [n]. Here, all relevant features such as consonantality, voicing, nasality and place are joined to the root node in a hierarchy of nodes and association lines.

The relationship between individual nodes on adjacent tiers is not, however, biunique. As shown in (4), a node dominated by one root node can be associated with a node dominated by another root node. Cases of assimilation are therefore characterized as the spread of nodes or features by means of reassociation. For instance, the place assimilation of a nasal to an immediately following [b] is as in (4), with the spread of the place node indicated by the dashed line. Notice particularly that it is not the separate features that are spreading, but rather the place node.

Although feature geometry has been used primarily for fully developed languages, 
(3) Feature geometry of [n]

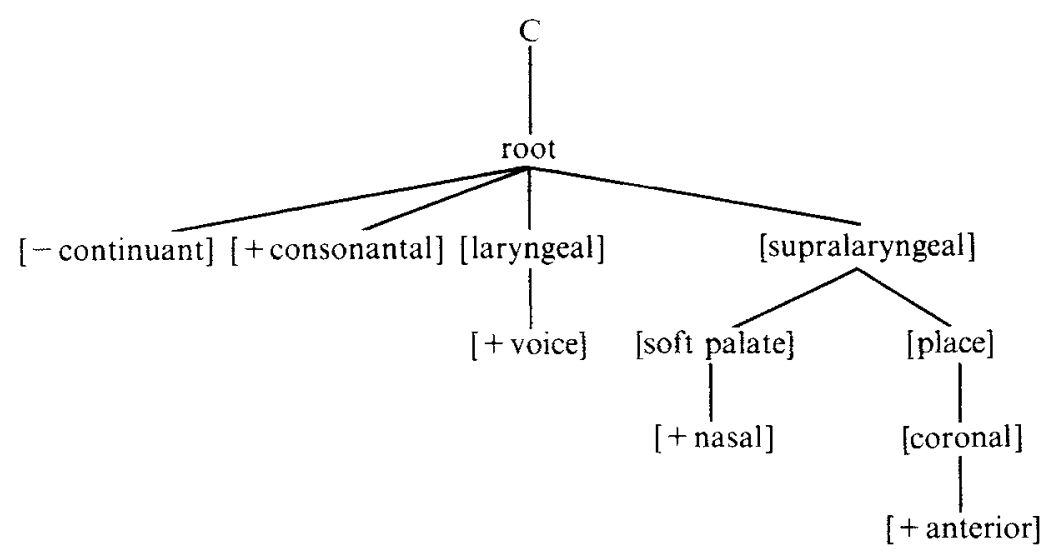

(4) Place assimilation of nasal to b by feature-spreading

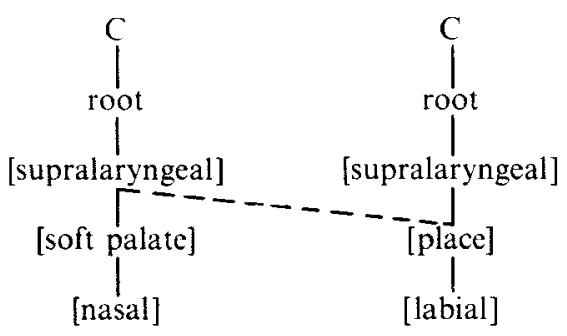

our investigations of the phonologies of misarticulating children indicate that this type of representation is useful for explaining various phenomena in those phonological systems. To demonstrate this, we will use data from the phonologies of misarticulators from our own studies.

\section{Subjects and methods}

The children described here are taken from a group of 40 subjects gathered for a larger study investigating their phonological knowledge and learning patterns. Before treatment, the 40 children ranged in age from $3 ; 4$ to $6 ; 8$. All had at least six sounds in error across three manner categories in the Goldman-Fristoe Test of Articulation (Goldman and Fristoe, 1986) but fell within normal limits on all other tests including those of hearing acuity (re: ANSI, 1970) and receptive vocabulary (Dunn and Dunn, 1981). Gross organic bases for misarticulation were provisionally ruled out using the Mason and Simon (1977) Orofacial Examination Checklist.

For purposes of phonological analysis, data were gathered by means of a 306item single-word probe adapted from Gierut (1985). Elicitation sessions were taperecorded and transcribed on-line by two independent transcribers. Discrepancies in transcriptions were resolved by consensus decision using the tape recordings. All data for the present study were fully resolved and elicited at a time prior to any treatment. 


\section{Discussion}

We will examine three representative rule-governed phonological phenomena to demonstrate the usefulness of feature geometry in the description of disordered phonologies. First, we will discuss coalescence phenomena; second, affricate/fricative alternations; and finally alveolar stop/glottal stop alternations.

\section{Coalescence}

A number of subjects (seven) in our study showed evidence of coalescence (see also Chin and Dinnsen, 1990), by which we mean that a target adult cluster is realized in the child's phonetic form as a single sound, with some features from one of the target cluster elements and some from the other. Examples of this are given in the table in (5).

\section{[5] Coalescences \\ Subject 10: [ftm] 'swim', [fip] 'sweep' \\ Subject 11: [由ipun] 'sweeping' \\ Subject 25: [feto] 'smell'}

In all cases, these coalescences occurred when the target cluster was made up of [s] plus a sonorant, and the sonorant was usually labial. The segment resulting from a coalescence was a voiceless labial fricative [f], or in one case $[\Phi]$. The occurrence of $[\Phi]$ for Subject 11 is an important case, because except for its presence as the realization of a coalesced cluster, this sound did not otherwise occur in the child's inventory. Therefore, this was not a case of simple substitution but rather a new sound created by the coalescence. All of the subjects who showed coalescences had mostly one-element realizations of target clusters, but there were always a few twoelement realizations. For this reason, we suggest that these children are at a stage of development where they are just beginning to realize target clusters with two elements. First to develop are the underlying representations, which are represented with two elements, but a phonological rule changes these to single coalesced elements in phonetic form. The problem for both place-voice-manner and distinctive feature systems is to derive a single sound from two underlying sounds. The rules that these systems need to use are circuitous and lack explanatory power, A feature geometry is uniquely suited to represent these coalescences by means of reassociation. First, the geometric structures of both target consonants are present in the child's underlying representation, as in the example in (6). Then a rule acts on this underlying structure, reassociating the first supralaryngeal node with the second place node, as indicated by the dashed line in (6). All superfluous structure, including the second $\mathrm{C}$ slot and all structure below it, is then deleted. The resulting coalesced structure appears in phonetic form as in (7).

\section{Affricate/fricative alternations}

A second illustration of geometry is alternation between affricates in word-final position and fricatives in intervocalic position; this kind of alternation was evident in the phonologies of a number of our subjects. Examples of this are given in (8). If we consider the affricates as underlying and the fricatives as derived, a feature 
[6] Child's underlying representation for coalesced segment (/sw/)

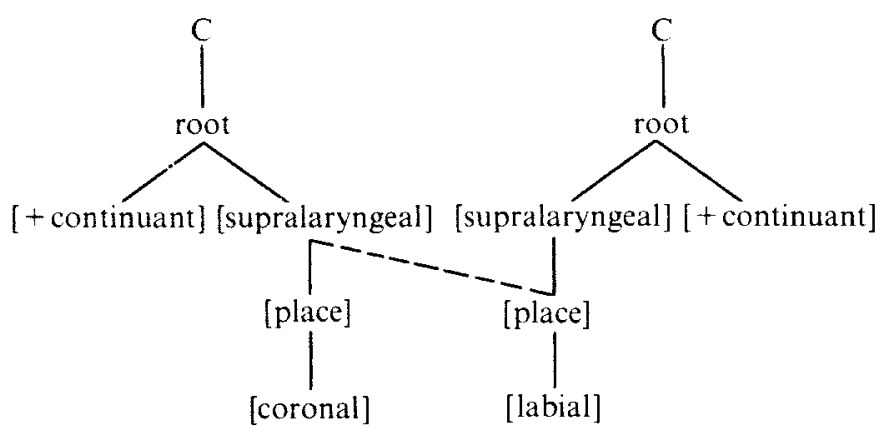

[7] Child's phonetic representation for coalesced segment ([f])

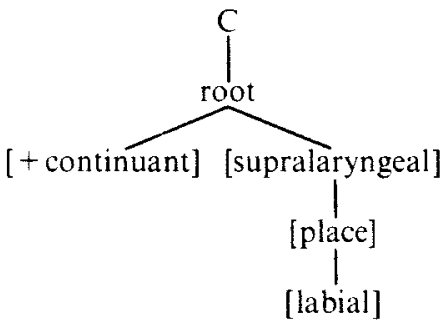

(8) Affricate/fricative alternations

$$
\begin{aligned}
& \text { Subject 14: [keıdð] 'cage' [keıði] 'cagey' } \\
& \text { [bwıdð] 'bridge' [bwıði] 'bridgey' } \\
& \text { [bædð] 'badge' [bæði] 'badgey' } \\
& \text { Subject 22: [wits] 'witch' [wisi] 'witchie' } \\
& \text { [wats] 'watch' [wasi] 'watchie' }
\end{aligned}
$$

system like the one proposed by Chomsky and Halle (1968: 317-318) accounts for this alternation by changing the feature [continuant] from minus for the affricate to plus for the fricative. The IPA system uses the same diacritic to transcribe both affricates and double articulations, that is, a top ligature over two different symbols. For the affricates, however, there is a definite temporal sequencing of the two elements that is not present in the double articulations. That is, although the two gestures in a double articulation are considered to occur simultaneously, in an affricate the stop portion always precedes the fricative portion. As illustrated in the figure in (9), in a feature geometry this is expressed by configuring the representation of the affricate so that a [-continuant] portion precedes the [+ continuant] portion in the geometry, with both of these dominated by a single root node to indicate the phonemic status of the affricate as a whole. In (9), the left-to-right ordering of [- continuant] and [+ continuant] indicates temporal sequencing on the same tier. The derivation of the fricative is now quite straightforward. Rather than using a feature changing rule as in distinctive feature systems, feature geometry simply 
(9) Representation of affricate in feature geometry

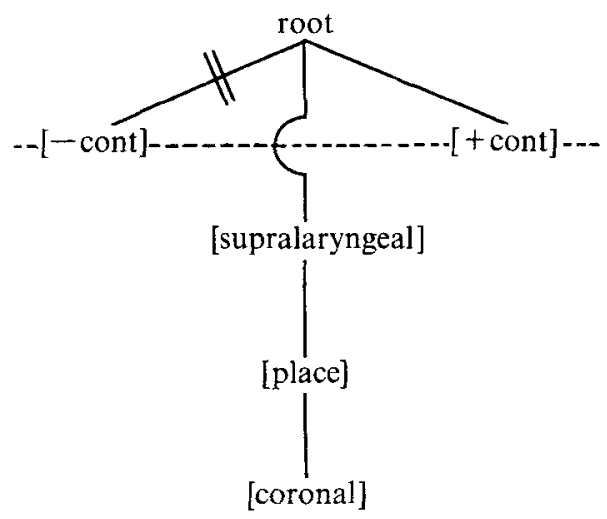

delinks the [-continuant] node from the root node, indicated in (9) by the crossedout association line. The remaining features, including [+ continuant], voicing, and the supralaryngeal features, all remain and describe fully the derived fricative.

\section{Alveolar stop/glottal stop alternations}

Our third illustration includes cases in which target $/ \mathrm{t} /$ showed alveolar and glottal alternations in forms such as those from Subject 35 given in (10). If we use a placevoice-manner system, we can describe this alternation as the change of an alveolar stop to a glottal stop word-finally, but although this is descriptively accurate, it nevertheless lacks explanatory adequacy. Likewise, using Chomsky and Halle's (1968) features, we can change the coefficients of the features [anterior], [coronal], [low], [consonantal], and [sonorant], but again, there is no theory-bound explanation of why exactly these features should change together.

(10) Alternations of alveolar stop and glottal stop (Subject 35)

$$
\begin{aligned}
& \text { [batrig] biting' [baı?] 'bite' }
\end{aligned}
$$

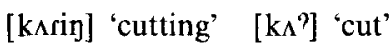

$$
\begin{aligned}
& \text { [irin] 'eating' [i?] 'eat' }
\end{aligned}
$$

As shown in (11), because the features that differentiate [ $[\mathrm{t}]$ from $\left[{ }^{?}\right]$ are all supralaryngeal features, feature geometry views the alternation as simply delinking of the supralaryngeal node and as an automatic consequence all the features subordinate to it. The features remaining, those outside the box in (11), exactly describe a glottal stop, that is, a voiceless non-continuant with no supralaryngeal articulations. $\dagger$

IIt has been pointed out to us, and quite correctly so, that production of the alveolar stop involves vocal fold abduction, whereas production of a glottal stop involves adduction. Our view is that a [- cont] specification will countenance, as a default, supralaryngeal articulation. Thus, because of coronal closure for the alveolar stop, the vocal folds can be abducted $([+$ spread glottis] $)$. A [ - cont] specification and a lack of supralaryngeal features, however, will require closure at some other place, so that the automatic consequence is vocal fold abduction $([+$ constricted glottis $])$. 
(11) Geometric representation of alveolar stop/glottal stop alternation

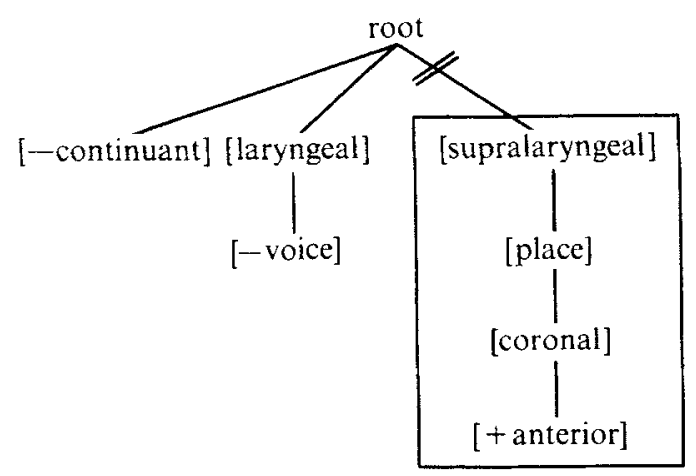

The feature geometry thus accounts for alveolar stop/glottal stop alternations by means of a single process, i.e. deletion of all supralaryngeal features. This is what renders these alternations relatively more plausible than, for example, alternations of [t] with [h]. The latter case would require changes at two sites, i.e. [cont] and the supralaryngeal node. Furthermore, we would not expect $[\mathrm{t}] /[\mathrm{h}]$ alternations in the absence of evidence of $[\mathrm{t}] /[\mathrm{s}]$ alternations and $[\mathrm{s}] /[\mathrm{h}]$ alternations as well.

\section{Conclusion}

To conclude, both place--voice-manner and distinctive feature systems have been used widely as descriptive and explanatory devices for the analysis of phonological systems, both normal and disordered, However, both types of systems also have disadvantages. Place-voice-manner systems appeal to large articulatory classes but have difficulty in describing subcategories and groups across categories. On the other hand, distinctive feature systems have no way of guaranteeing that certain groups of features may act together in particular rules. We believe that feature geometry, by providing for both the individual and grouped behaviour of features, overcomes these problems and reconciles the large category nature of place-voice-manner systems and the smaller unit characteristics of distinctive feature systems. We hope that continued research of this sort will reveal insights about the nature of phonological developments and variation.

\section{Acknowledgements}

We are grateful to Phil J. Connell, Mary Elbert, Judith A. Gierut, and two anonymous reviewers for their helpful comments during the course of this research. A version of this paper was presented at the American Speech-Language-Hearing Convention held in Seattle in 1990. This work was supported in part by grants to Indiana University from the National Institutes of Health, Nos. NS20976 and DC00260. 


\section{References}

American National Standards Institute. (1970) Specifications for Audiometers. (ANSI S3.6-1969) (New York: ANSI).

CHIN, S. B. and DINNSEN, D. A. (1990) Consonant clusters in disordered speech: correspondence strategies and constraints. Paper presented at the Fifteenth Annual Boston University Conference on Language Development, Boston, Massachusetts.

Chomsky, N. and Halle, M. (1968) The Sound Pattern of English (New York: Harper \& Row).

Clements, G. N. (1985) The geometry of phonological features. Phonology Yearbook, 2, $225-252$.

Duckworth, M., Allen, G., Hardcastle, W. and Ball, M. (1990) Extensions to the International Phonetic Alphabet for the transcription of atypical speech. Clinical Linguistics and Phonetics, 4, 273-280.

Dunn, L. M. and Dunn, L. M. (1981) Peabody Picture Vocabulary Test-Revised (Circle Pines, Minnesota: American Guidance Service).

GIERUT, J. A. (1985) On the relationship between phonological knowledge and generalization learning in misarticulating children (Unpublished $\mathrm{PhD}$ dissertation, Indiana University).

Goldman, R. and Fristoe, M. (1986) Goldman-Fristoe Test of Articulation (Circle Pines, Minnesota: American Guidance Service, Inc).

Goldsmith, J. A. (1990) Autosegmental and Metrical Phonology (Oxford: Basil Blackwell).

Ladefoged, P. (1990) Some reflections on the IPA. UCLA Working Papers in Phonetics, 74, $61-76$.

Mason, R. M. and Simon, C. (1977) An orofacial examination checklist. Language, Speech and Hearing Services in Schools, 8, 155-163.

McCarthy, J. J. (1988) Feature geometry and dependency: a review. Phonetica, 45, 84-108.

SAGEY, E. C. (1986) The representation of features and relations in non-linear phonology (Unpublished $\mathrm{PhD}$ dissertation, Massachusetts Institute of Technology). 